\title{
A INDETERMINAÇÃO DE SENIOR: PERSPERCTIVAS E REALIZAÇÕES DE UM PROGRAMA DE PESQUISA*
}

\begin{abstract}
Antonio Maria da Silveira**
"Exatamente essa que constitui decerto a sabedoria humana [...]: em não julgar saber o que de fato não sei."

Apologia de Sócrates, Platão
\end{abstract}

\begin{abstract}
O tema central deste programa de pesquisa pode ser ligeiramente colocado nas palavras de Marshall, como citado em GROENEWEGEN (1990, p. xiv):
\end{abstract}

A Economia Política raramente responde qualquer questão social, assim como qualquer questão social raramente pode receber respostas independentemente da Economia Política.

Passo a uma elaboração sucinta da formulação a que cheguei depois de um esforço para bem vincular à literatura econômica as análises precursoras. Elas vinham então sendo predominantemente escritas a partir da observação direta e da vivência do fenômeno, como exposto abaixo.

Existe uma divisão de trabalho nas ciências em geral, qualquer que seja o campo de conhecimento. Tanto em ciências da matéria inerte quanto nas ciências da vida, ou da sociedade, a passagem do tempo tende a estabelecer uma especialização em, pelo menos, três esferas científicas do saber, além do conhecimento prático.

Primeiro, o nível de abstração maior da ciência pura ou abstrata, com teorias indiretamente aplicáveis. Como exemplos, Física e Economia Pura ou

* Apresentado em mesa especial com que o autor foi distinguido no II Encontro de Economia da Região Sul, Curitiba, 27-28/9/1999. O autor usa livremente aqui suas publicações anteriores. JEL: B00 Metodologia e História do Pensamento Econômico. Palavras-chave: Vício Ricardiano, Aplicabilidade de Teorias, Realismo, Economia Positiva, Economia Normativa, Economia Pura ou Abstrata, Economia Social ou Aplicada, Arte da Economia, Contenda do Método.

** Professor Titular da UFRJ (aposentado). 
Abstrata (Teoria Neoclássica da Firma, Teoria do Crescimento). Para este nível, valem as palavras de SENIOR (1938, p. 3):

Mas as conclusões dele [do Economista Político], não importando a generalidade ou verdade que encerrem, não o autorizam a adicionar uma simples sílaba de conselho [...] A tarefa do economista político é [...] estabelecer os princípios gerais cuja ignorância seria fatal na condução dos afazeres práticos; não é, contudo, aconselhável, nem talvez praticável, entender tais princípios como guias exclusivos ou, mesmo, como guias mais importantes [nos afazeres práticos]...

Segundo, o nível de abstração menor da ciência aplicada, com teorias diretamente aplicáveis sob qualificações freqüentemente severas. Como exemplos correspondentes, ciências da engenharia e economia social ou aplicada (Teoria Comportamental da Firma, Teoria do Desenvolvimento Econômico). Para este, tomamos as palavras de KNIGHT (1960, p. 111):

Para dizer agora um pouco mais sobre o irrealismo da teoria econômica pura [...] Todas as ciências do homem e da sociedade estão envolvidas quando se pressiona mais e mais a questão [da ação social, da escolha pública e da decisão política] - particularmente história e, possível e até mais especialmente, ética.

KNIGHT segue MILL (1877, p. 152):

Cada arte pressupõe, não uma ciência, mas ciência em geral; ou, pelo menos, muitas ciências distintas.

MILL segue SENIOR (1938, p.3):

Decidir em cada caso até quando as conclusões do Economista Político devem gerar ações, pertence à arte de governo, arte para qual a Economia Política é apenas uma das muitas Ciências subservientes.

Fora da ciência está o nível de trato com a realidade, ou nível prático, que se espera informado pelo conhecimento teórico. Como exemplos, engenheiro ou economista profissional (assessor de estratégia empresarial, assessor de ministro do planejamento). Para este, continuo nas palavras de MILL (1877, p. 155):

Ninguém que busque estabelecer proposições para orientação da humanidade pode dispensar, não importando suas realizações científicas, o conhecimento prático sobre as maneiras em que afazeres do mundo são de fato conduzidos, e uma ampla experiência pessoal com idéias, sentimentos, e tendências intelectuais e morais de fato existentes em seu país e em sua própria época.

Em princípio, ou por definição, especificidades não são captáveis por 
teoria alguma. Dada a importância que elas tendem a assumir numa ocorrência qualquer do fenômeno social, teorias podem não explicar casos particulares.

A prática requer ainda opções de valor, além dos juízos de valor implícitos ou explícitos nas próprias teorias. Num exemplo, a política econômica numa opção Brasil-potência, como no recente período do autoritarismo, difere significativamente da política numa opção alternativa de Brasil-humanismo ou, igualmente, de Brasil-dependentismo.

Finalmente, os trabalhos acadêmicos de posicionamento, em políticas públicas ou empresariais, enquadram-se usualmente na fronteira entre a prática e a arte da ciência. Mas nitidamente na arte estão a história econômica, incluindo naturalmente a história contemporânea, e muitos estudos de caso, em particular os que visam aperfeiçoar teorias.

Tem-se o terceiro e menor nível de abstração da ciência, no qual se dá o entrelaçamento do saber universal com o específico. SCHUMPETER (1986, p. 812-813) diz magistralmente sobre essa forma do saber:

Não devemos esquecer que, embora a pesquisa [histórica monográfica], mais o estudo coordenado de seus resultados, nunca venha a produzir teoremas articulados, ela pode realizar, para mentes apropriadamente condicionadas, algo que é muito mais valoroso. Ela pode gerar uma mensagem sutil, transmitir um entendimento íntimo dos processos sociais ou especificamente econômicos, um senso de perspectiva histórica ou, se preferem, a coerência orgânica das coisas, cuja formulação é difícil ao extremo, talvez impossível.

Atualizando e generalizando os economistas citados, que se constituem em marcos do desenvolvimento da matéria, formulo o que chamo Indeterminação de Senior nos termos seguintes:

As proposições da economia pura, não importando a generalidade ou verdade que encerrem, não autorizam conclusões normativas, mas não podem ser ignoradas. A economia social positiva entrelaça teorias econômicas puras e todas as ciências sociais, mas com relevância variável. Conclusões normativas - sob a forma do que não pode ser feito são deriváveis de proposições da economia social, mas são ainda qualificáveis pelas especificidades de cada caso.

A indeterminação realça a multiplicidade de formas complementares do conhecimento científico e aponta as limitações de cada uma delas. A indeterminação é o que se encontra inobservado no hábito que SCHUMPETER (1986, p. 540/1171) identificou e chamou de Vício Ricardiano:

Eles [Senior, Mill e outros] quiseram apenas dizer que as questões de política econômica envolvem sempre tantos elementos não-econômicos, que seu tratamento não deve ser feito na base de considerações puramente econômicas [...] poder-se-ia apenas desejar que os econo- 
mistas daquele (como de qualquer outro) período nunca se esquecessem deste toque de sabedoria - nunca fossem culpados do Vício Ricardiano [...]

[...] o que temos chamado acima de Vício Ricardiano, isto é, o hábito de empilhar uma carga pesada de conclusões práticas sobre uma fundação tênue, que não se lhe iguala, mas que parece, em sua simplicidade, não apenas atrativa, mas também convincente.

As palavras de SCHUMPETER, dentro do contexto de acusações contra os economistas no Brasil, e no mundo, apontam para uma justificativa social do programa de pesquisa ${ }^{1}$. Cabe investigar os males de que são acusados e buscar soluções. A análise da indeterminação e do vício impõe-se assim como questão maior.

Outra justificativa está no entendimento do "atraso" relativo da economia como ciência. Atribui-lo à categoria de "ciência nova" é duvidoso, bastando lembrar que Willian Petty foi veterano de Newton na Royal Society (HAYEK, 1975, p. 252). Atribui-lo à complexidade e à mutabilidade do fenômeno, assim como à natureza semi-experimental do saber, é uma das meias-verdades em análise.

Atribui-lo à inexistência do nível de divisão de trabalho alcançado nas ciências da matéria inerte e da vida é outra das meias-verdades sob investigação. Aqui, o Vício Ricardiano é indicador da recusa do especialista no reconhecer as limitações de sua especialização.

Faltaria ao economista puro, teórico ou econometrista, o senso científico do físico, teórico ou experimental? Faltaria ao economista prático o preceito ético do clínico geral, "que não trata de doenças, mas de pacientes"? Faltaria ao público reconhecer a diferença entre o economista puro e o social? A importância da divisão de trabalho para o progresso é, naturalmente, matéria sabida de todos, desde Adam Smith que a deixou bem clara.

As perguntas evocam questões sobre a formação dos economistas, como cientificismo (HAYEK, 1975, p. 249) e cegueira científica-KUHN (1971, p. 37/61) usa a expressão e fundamenta-se em Piaget e na Gestalt; pode-se alternativamente argumentar, sem perda, em termos de ideologia científica, como SCHUMPETER (1949, p. 348) derivou de Marx. A falta de instrução filosófica nos currículos escolares é patente.

Evocam, ainda, questões maiores, como grau de tecnocracia mais acentuado nos economistas, causado talvez por irrecusável chamamento do dever

${ }^{1}$ Grandes literatos escreveram romances que são verdadeiros libelos contra a comunidade, como A Vigésima-Quinta Hora, de Gheorghiu, ou já no século passado, Hard Times, de Charles Dickens. 
público, como interpreta SCHUMPETER (1949, p. 346). Evocam também questões éticas, como comportamento interesseiro, o qual é possivelmente aguçado pelo fato de se constituir na premissa em que se fundamenta, em muitos departamentos, todo o ensino da ciência: o Homem Econômico.

Passo a uma elaboração sobre as linhas de pesquisa em que o programa vem sendo desenvolvido. Indico o feito e exemplos mais promissores do que se tem a fazer. Noutras palavras, minha intenção aqui é renovar o projeto de pesquisa e minha tese é que sua fertilidade aponta para outra década de trabalho.

\section{LINHAS DE PESQUISA DO PROGRAMA ${ }^{2}$}

\section{Conexão com a História do Pensamento Econômico}

Uma linha bem acadêmica, fértil e mais acessível para teses de mestrado e monografias de graduação, visa continuar a conexão do tema à literatura econômica.

Um objetivo seria investigar a extensão em que grandes economistas se colocam em perspectivas compatíveis com a indeterminação, ou se mostram adictos do Vício Ricardiano (LOPES, 1992; LIBÂNIO, 1997). A pesquisa é mais fértil quando focaliza problemas ou controvérsias que se dissolvem com o reconhecimento da especialização ou divisão de trabalho acadêmico.

Como exemplo, SCHUMPETER sugere que a indeterminação foi esquecida em virtude do desvio de atenção para o problema de juízos de valor; Cairnes, Sidgwick e WEBER o teriam provocado. A questão e os autores constituem-se em temas excelentes de pesquisa, assim como o próprio Ricardo, ou KEYNES, casos estes típicos do vício, na avaliação de SCHUMPETER (VERNENGO, 1993a, AMARAL, 1995, ORIND, 1995).

Pesquisas ambiciosas envolveriam autores como Adam Smith ou um metodólogo maior, NEVILLE KEYNES (1904). HUTCHISON (1981) seria outra alternativa. Os dois últimos escreveram sobre a Contenda do Método, constituindose na seqüência natural de SILVEIRA (1992e), minha primeira incursão na história do pensamento econômico alemão. No caso japonês, Uno Kozo é de interesse maior, pois aborda o marxismo ao longo das três esferas de abstração e parece, mesmo, acusar o Vício Ricardiano no próprio Marx (MORRIS-SUZUKI, 1991, p. 118).

Quanto a Smith, ocorre-me uma verificação sobre a importância, em sua obra, das aspirações crescentes do ser humano. Trata-se de uma dimensão

${ }^{2}$ Os trabalhos realizados pelos alunos e por mim estão listados, antes da Bibliografia, nas categorias Trabalhos Realizados, Trabalhos Orientados e Trabalhos Precursores. 
econômica do comportamento não captada no homem econômico, mas recuperada na unidade analítica da economia comportamental de SIMON (1955), o homem administrativo. A ausência dessa dimensão na teoria neoclássica (economia pura) limita o entendimento dos processos de crescimento, numa extensão que necessita ser estudada.

\section{Análise Comparativa entre Economia Pura e Social}

Uma linha que tende a ser mais complexa foi iniciada em SILVEIRA (1992a), artigo que estabelece paralelo entre uma teoria pura (micro-neoclássica) e outra socioeconômica (estratégia empresarial) - veja também Garcia DUARTE (1993, 1994).

Um bom problema: o neo-austríaco LACHMANN (1973) criticou a macroeconomia em termos do seu despropositado grau de abstração. Seria uma teoria pura, se fosse interpretada no contexto da indeterninação; por mais estranho que pareça, sua argumentação não pode ser ignorada. Tomando o neo-austríaco HAYEK (1975), entretanto, a macro é economia correlativa (MARGENAU 1966, p. 29-30), além de cientificismo. Para HICKS (1980) por outro lado, a macro é da socioeconomia. O estabelecimento de fundamentos microeconômicos da macro vem, certamente, em suporte dos dois últimos.

Um problema excelente seria o estudo da teoria do crescimento como versão pura da teoria socioeconômica do desenvolvimento - o marco de separação entre ambas é a década de cinqüenta (BLAUG, 1985, p. 95). Para que se aquilate a importância do estudo, basta uma proposição aparentemente polêmica: exigir, em trabalhos ou programas de pós-graduação voltados para desenvolvimento, a utilização de modelos recentes de crescimento - uma postura comum entre economistas puros, filosoficamente desinformados - é inviabilizar a pesquisa em desenvolvimento econômico.

Dissolve-se a polêmica, a menos de cegueiras científicas, quando se observa o paralelo na física e na ciência da engenharia - a postura condenaria a última à extinção. Permanece, entretanto, a pergunta do quanto se exigir de economia pura (física) na economia social (engenharia). Tomando LAKATOS, a indicação para a resposta estaria nos núcleos duros, acessíveis em livros-texto de introdução, deixando de fora os cintos protetores, nos quais se desenvolve o altamente perecível trabalho de fronteira dos programas de pesquisa pura.

\section{Linguagens da Economia Pura e Social}

Em primeira aproximação, se a lógica predomina nas teorias puras, a dialógica domina as teorias socioeconômicas e a arte da economia. Dialógica é o termo aqui usado para substituir a lógica quântica, como em HEISENBERG (1963, p. 156-157), ou a dialética, como em GEORGESCU-ROEGEN (1967, p. 23-24), ou ainda a lógica da probabilidade ou da linguagem natural, como em 
KEYNES (CARABELLI, 1988, p. 141-142):

Keynes enfatizou a necessidade da probabilidade ser aberta, e não como linguagens artificiais, as quais são compostas por um número finito de membros, com alternativas exaustivas [...] Além disso, ele descreveu sua lógica da probabilidade como não obedecendo ao princípio da não-contradição [...] Keynes havia já observado que 'a lógica da probabilidade é da maior importância, porque é a lógica do discurso ordinário, através do qual as conclusões práticas de ações são geralmente alcançadas'.

CARABELLI (1988, p. 282) especula sobre a correspondência entre essa lógica e a retórica da economia em MCCLOSKEY (1983), no que certamente se iguala ao último em ousadia de hipóteses. Um ordenamento do discurso em termos dos três níveis de abstração, seguido de distinções dentro de cada nível, como o da ciência pura normal e extraordinária (revolucinária) de KUHN (1971), seria investigação que se sugere extremamente fértil.

\section{Filosofia da Ciência}

A conexão com a filosofia geral da ciência torna-se indispensável na medida em que o programa se afirma. A indeterminação lembra Aquino (cristianismo) que, no seguir Maimônides (judaísmo) no seguimento de Averrois (islamismo), construiu a super-estrutura que legitimou o desenvolvimento da ciência: especificidades não são captáveis por teoria alguma, proposições gerais não resolvem casos particulares. De Aquino ao realismo, tem-se uma linha para articular a Indeterminação de Senior à literatura filosófica.

Mais atrativa, talvez, seria a vinculação mais estreita do programa com filósofos da ciência de maior destaque recente, como POPPER, KUHN, LAKATOS e FEYERABEND (GRUMBACH, 1997). Por outro lado, o corte da literatura filosófica na tricotomia pura-aplicada-arte tem efeitos tão esclarecedores e desconcertantes quanto a dicotomia ciência normal-extraordinária de KUHN, prometendo assim grande fertilidade. Sigo com exemplos de questões, numa formulação ousada e polêmica.

A dicotomia kuhniana coloca POPPER num posicionamento paradoxal. Suas conjecturas e refutações, sua lógica formal, sua conceituação de testes e erros etc., foram escritas para a ciência extraordinária, mas permanecem válidas apenas para períodos normais, cuja existência ele reconhece mas abomina. Para KUHN, pelo contrário, períodos normais servem de demarcação do conhecimento como ciência.

Estendendo o referencial da indeterminação para o domínio filosófico, POPPER aparece como filósofo puro, KUHN como aplicado, mas ambos mostram-se, à primeira vista, vítimas da cegueira científica ao não se reconhecerem como tais - houve, pelo menos, uma ocasião que exigia o reconhecimento: o 
simpósio em que se esforçaram por conjugar seus trabalhos; observa-se, no entanto, que ignoraram por completo seus distintos níveis de abstração (LAKATOS; MUSGRAVE, 1980). Ironicamente, a cegueira científica é peça fundamental para a teoria de KUHN!

Há mais, KUHN não apenas ignora a ciência aplicada, com sua hipótese de evolução autônoma do conhecimento puro, mas parece desmerecê-la quase tanto quanto POPPER. Há que também se investigar algo do mesmo desmerecimento que transparece na visão de ambos a respeito das ciências sociais, apesar do uso extenso que fazem delas em suas próprias pesquisas.

Noutra questão, vale transcrever uma das citações que selecionei de KUHN (1980, p. 16) não só para sugerir sua posição aplicada, mas porque leva à dialógica, à lógica da linguagem natural de KEYNES, em uma das matérias que mais requer da filosofia para a compreensão dos fundamentos e da aplicabilidade das teorias econômicas:

Ao levantar essas questões, meu objetivo é sugerir que, embora a lógica seja um instrumento poderoso e em última instância essencial para a investigação científica, pode-se ter conhecimento seguro sob formas em que ela não é bem aplicável. E mais, quero sugerir que a articulação lógica não é um valor em si mesma, devendo ser empreendida somente quando as circunstâncias o exigirem, e na extensão em que o fizerem.

Na dicotomia kuhniana, a teoria pura não se restringe à lógica, pois os períodos revolucionários - as escolhas entre teorias conflitantes - exigem a persuasão em termos dos valores da comunidade científica. A persuasão, com seu caráter não imperativo, é da linguagem dialógica (ao manter a lógica como linguagem predominante da teoria pura, entretanto, estou afinado, com o mesmo KUHN, na importância maior que atribui aos períodos normais).

\section{Análise de Revoluções Científicas na Economia}

O dissenso entre economistas é consenso folclórico, encontrando causas aventadas em, por exemplo, SCHUMPETER (1949, p. 345-346) e ROBINSON (1983, p. 27-28), e evidências empíricas corroboradoras, como em FREY et al. (1984). Ao conflito paradigmático no interior de cada escola de pensamento, como entre físicos durante as revoluções, acrescenta-se o conflito entre escolas, particularmente quando diferenciadas pelo nível de abstração do conhecimento.

Assim, a perspectiva da indeterminação permite desembaralhar muitas questões, vendo, por exemplo, SIMON, e seus seguidores da economia comportamental, numa versão aplicada da teoria neoclássica; esta iluminou basicamente a construção daquela, havendo complementaridade, bem mais do que substitutabilidade entre ambas.

Por outro lado, a perspectiva econômica pode também esclarecer várias questões da filosofia da ciência, como procuro exemplificar em meus estudos da 
revolução da escolha pública (SILVEIRA, 1990a, 1990b), particularmente no primeiro deles.

Proponho que na questão da escolha entre teorias conflitantes, a economia, e KEYNES em particular, deve iluminar a filosofia, talvez mais do que o esperado oposto. Tem-se um vastíssimo campo de pesquisa, nada menos do que a economia do conhecimento em complemento à sociologia do conhecimento.

\section{Tecnocracia}

O Vício Ricardiano alimenta uma tendência não democrática talvez crescente: a tecnocracia como versão moderna da teocracia - perdoem-me os leitores se me sinto na necessidade de afirmar que tecnocrata não é sinônimo de técnico, que "economistas no governo" não significa tecnocracia.

A indeterminação, no minar a postura tecnocrática, é uma grande questão, controvertida e de significado maior. Mantenho muita expectativa na conexão deste estudo com a questão geral do poder dos peritos e dos peritos no Poder - veja HASKELL (1984) e PRICE (1965).

Como exemplo da importância da pesquisa nessa dimensão, o entendimento de posições ministeriais como técnicas, quando genuíno, é tecnocracia - quando usado como arma no embate político, não passa naturalmente de mera retórica. Quando um professor se torna ministro, sem passagem pela política, a perspectiva da indeterminação aponta "iminentes perigos".

Se se trata de um economista puro, abstrato, pesquisando na fronteira do conhecimento, a tendência será cuidar apenas do lado econômico da realidade (Vício Ricardiano, SCHUMPETER; cegueira científica, KUHN) e usar a sociedade como cobaia (aplicação direta de inovações teóricas insuficientemente testadas, LAKATOS).

O ponto lembra HAYEK, como citado por LUNDBERG (1975, p. 242), "um economista que é apenas um economista tornar-se-á provavelmente um incoveniente, se não um iminente perigo". O perigo tende a ser menor no caso do economista social, mas ainda lhe faltam as especificidades, pois seu trabalho acadêmico também exige que elas sejam sistematicamente ignoradas, dando atenção exclusiva aos fatores universal e necessariamente presentes nas ocorrências do fenômeno.

Os iminentes perigos decrescem mais para o acadêmico do estudo de caso (MYRDAL) e da história econômica (Schmoler), desde que sejam capazes de tratar o fenômeno econômico sob a iluminação de todas as ciências sociais, como o eram os exemplos indicados entre parênteses. Permanece aqui, entretanto, o problema da cegueira científica, como observável no próprio Schmoler, na Contenda do Método (SILVEIRA, 1992e). 
Permanece também o fato de que, na medida em que se fala de um bom cientista, não importando se no nível puro, aplicado ou na arte, seu "saber como" tende a estar na pesquisa e no ensino apenas, o que nada obviamente tem a ver com a prática ministerial. Há que se ressalvar em tudo, entretanto, que se tratam de proposições sobre tendências apenas. A própria perspectiva da indeterminação realça enfaticamente que cada caso é um caso.

\section{Formação do Economista}

KNIGHT parece acusar os cientistas sociais de terem menor conhecimento do ser humano e da sociedade do que escritores, filósofos e pensadores religiosos (SILVEIRA, 1994). Apresento duas hipóteses para estudo: a incompletude do conhecimento científico como percebida na indeterminação de Senior é certamente uma; a outra é a cegueira científica.

Pode-se muito pesquisar sobre a formação e o trabalho dos economistas, buscando as causas do Vício Ricardiano e de outros problemas da comunidade. Numa questão ética maior para a academia estadunidense, existem evidências estatisticamente significativas no sentido de que a pós-graduação em economia, pelo menos quando exclusivamente neoclássica (homem econômico), acentua o comportamento interesseiro dos alunos (FRANK et al., 1993).

Numa questão pragmática, a chamada Comissão Krueger (KRUEGER, 1991, p. 1035-1053) buscou analisar a crescente perda, pelos recém-doutorados nos Estados Unidos, do mercado em organizações não-acadêmicas e nos departamentos aplicados, como administração e políticas públicas (mais de 50\% dos empregadores). Formada pela Associação Estadunidense de Economistas, a Comissão foi composta por doze professores, dentre eles Arrow, Prêmio Nobel de 72, e Lucas, que veio a ser o laureado de 95.

Reconhecendo como verdadeira a queixa maior dos empregadores, a incapacidade de relacionar teoria e prática, a Comissão chegou a chamar os recémdoutorados de "doutos idiotas". Considerando a divisão de trabalho nos termos da indeterminação, contudo, não há sentido em exigir do economista puro, especializado na transmissão e construção de teorias hipotético-dedutivas, um bom desempenho naqueles mercados. A formação de profissionais e de cientistas aplicados é do campo da economia social, em paralelo com a ciência da engenharia (SILVEIRA, 1992c, 1997).

No caso da graduação no Brasil, a Resolução CFE n. ${ }^{\circ}$ 11/84 estabelece o currículo mínimo claramente em economia social, como bem o demonstra o Parecer CFE n. ${ }^{\text {o } 375 / 84 ~(M E N D E S, ~ 1997) . ~ A ~ o b s e r v a c ̧ a ̃ o ~ d o ~ c u r r i ́ c u l o, ~ s e g u n d o ~}$ a perspectiva da indeterminação, mostra que não há qualquer perigo do Vício Ricardiano originar-se nessa formação quando bem ministrada (SILVEIRA, 1995, 1996). 


\section{CONCLUSÃo}

Parece-me apropriado concluir com a revisão de alguns dos problemas e soluções que me levaram à Indeterminação de Senior. Primeiro, apropriado porque devo reconhecer em meu próprio trabalho que a história importa. A forma em que o conhecimento é desenvolvido diz muito sobre sua plausibilidade, como defende SIMON (1968, p. 458), ao contrário de POPPER.

Segundo, porque, concordando agora com POPPER (1978, p. 13-15), problemas e soluções temporárias constituem-se em método de geração do conhecimento. Há uma terceira razão, bem expressa nas palavras de MYRDAL $(1969$, p. 6) e certamente mais importante por mais que se discorde dele:

Estes pensamentos não são universalmente aceitos. Pelo contrário, a metodologia da ciência social é, em grande parte, metafísica e pseudoobjetiva. Pode não ser assim inteiramente fora de ordem - e indevidamente auto-centrado - esquematizar a rota que o pesquisador individualmente seguiu para alcançá-los.

Um problema inicial foi a sensação do nada saber, que me incomodou ao término de três cursos, Engenharia (1963), Administração (MS/1968) e Economia (PhD/1971). Estudei com afinco em boas escolas. Logo, a sensação era um problema, cuja solução só me ocorreu ao lembrar-me dele quando tive que escrever um memorial (SILVEIRA, 1992b). Boas escolas lecionam boas teorias, deixando de fora as especificidades; sem estas, a sensação tende a ocorrer, a não ser que uma explicação epistemológica seja passada ao estudante.

Como docente de uma escola integrada no campo da matéria inerte, Instituto Tecnológico da Aeronáutica (ITA 1964/66, 1971/74), e estudante de outra no campo social, Carnegie-Mellon University (C-MU 1966/71), tive um mesmo problema: o que faziam juntos, se divergiam tanto, seus cientistas puros e aplicados? Senti profundamente esse conflito paradigmático.

Permitam-me um toque de especificação do caso C-MU: tinha uma grande admiração pelos professores Allan H. Meltzer (meu orientador), Herbert A. SIMON (desenvolvi o hábito de ler seus trabalhos para recuperar-me das exigidas leituras chatas), e H. Igor Ansoff.

Não podia simplesmente aceitar os rótulos desabonadores alternadamente afixados às espécies distintas de pesquisa que faziam - meus sentimentos já estavam bem expressos por WAGNER (1886, p. 114-115), na sua análise da Contenda do Método, quando os mesmos rótulos eram também utilizados (SCHUMPETER, 1986, p. 814-815)!

Quando finalmente vim a ler KUHN (1971), fi-lo sofregamente na expectativa de superar a solução precária a que havia chegado (ocorreu lá pelo final da década de 70). Antes disso, um esforço contribuiu para aquela solução 
temporária. Foi uma análise, que empreendi a pedido do então Reitor do ITA, do primeiro Plano Básico de Desenvolvimento Científico e Tecnológico (PBDCT).

Percebi que o plano estava voltado para a prática, no pouco que tinha de ciência aplicada, e que de ciência pura nada continha. Depois de outras considerações, conclui que era prejudicial para as universidades, apesar de apresentá-las como principais beneficiárias (SILVEIRA, 1974).

Ao criticar o PBDCT, já me encontrava avançado no estudo de TOYNBEE (1962), o qual, apesar de inacabado, mudou significativamente minha visão de mundo. Meu objetivo era modesto. Buscava tornar-me historicamente bem informado, esforço retomado apenas em 1989, com TOYNBEE (1976).

Minha tese de doutoramento foi um trabalho econométrico sobre a inflação no Brasil. Ocorreu que, em 1973, os índices de custo de vida foram ostensivamente falsificados. Do ponto de vista acadêmico, o problema era a compreensão da natureza dos dados econômicos, solução só conseguida com a leitura de MORGENSTERN (1963), lá pelo final dos anos setenta (SILVEIRA, 1984a/b).

Antes disso, voltei-me para a pesquisa teórica. Entendia como falta de integridade intelectual continuar no trabalho econométrico, quando só podia partir de dados possivelmente falsificados, em extensão desconhecida. A observação de economistas no governo criou-me outro problema, dado que não agiam de acordo com as teorias que diziam professar. O problema acentuou-se quando também assumi a economia na prática, como consultor no setor privado.

A solução foi se me afigurando como pequenez da ciência econômica. Caracterizava-se como tal, temporária mas nitidamente, nas ocasiões em que mais me valiam os parcos conhecimentos de administração e de história. Buscava a solução na filosofia da ciência, quando li GEORGESCU-ROEGEN (1967).

Voltei-me para a filosofia econômica ao invés de abandonar a economia - estava começando a confundi-la com o que me parecia charlatanismo de muitos e era-me difícil continuar a vê-la como ciência. GEORGESCU introduziu-me a HEISENBERG (1963) e KNIGHT (1936), proporcionando-me o estímulo fundamental para assumir o ensino da filosofia econômica.

Dada a dificuldade dos alunos com o inglês dessa literatura, busquei recursos para traduzir boa parte de minha lista de leitura no princípio da década de 80 e publicá-la, depois de minha revisão, nas Edições Multiplic (EM), entre outubro de 1980 e dezembro de 1982. Continuei a fazê-lo enquanto na chefia do Conselho Editorial da Revista Brasileira de Economia (RBE), entre julho de 1983 e junho de 1985, mas apenas com as Conferências Nobel - indico sucintamente essas referências na bibliografia.

Consegui então um esquema de solução na forma da Indeterminação de Senior (SILVEIRA, 1984 a 1988), mas foi apenas com a leitura de 
SCHUMPETER (1986), que me introduziu a MILL (1877) e SENIOR (1938), que senti meu trabalho minimamente vinculado à literatura econômica.

Passei isoladamente por um longo processo de "revolução científica", se assim posso expressar-me, apesar de seu caráter individual. As anomalias foram sendo constatadas na prática, particularmente no seguimento do estado da economia e da política econômica.

Não passei, contudo, de minha posição neoclássico-monetarista para outra escola de pensamento, como costuma acontecer ao final de tais processos. Passei à crítica de todas as escolas e, mais tarde, à aceitação de todas, mas como meias-verdades temporárias.

Foi, em última instância, a necessidade de compreensão da política econômica, particularmente a de curto prazo, que me levou à filosofia. A Indeterminação de Senior emergiu como solução para um problema na prática da economia. Penso assim que trago um testemunho para a importância prática do conhecimento de metodologia e de história do pensamento.

\section{TRABALHOS REALIZADOS}

SILVEIRA, Antônio Maria da. The public choice sediton: variations on a theme by Buchanan. Cambridge: Clare Hall, University of Cambridge, 1989. Mimeog. (Em inglês apenas).

The Public Choice Sediton: variations on the theme of scientific warfare. Cambridge: Clare Hall, University of Cambridge, 1990a. Mimeog. Ensaio Econômico, Rio de Janeiro, n. 165, 1990. ENCONTRO NACIONAL DE ECONOMIA, 18., 1990, Brasília. Anais... Brasília: ANPEC,1990. v. 1, p. 147-166. Versão em português: A sedição da escolha pública: variações sobre o tema de revoluções científicas. Revista de Economia Política, n. 15, p. 37-56, jan./mar. 1996. Reproduzido em REGO, J. M. (Org.). Retórica na Economia. São Paulo: Editora 34, 1996.

. The public choice perspective and Knight's institutionalist bent. Cambridge: Clare Hall, University of Cambridge, 1990b. Mimeog. Ensaio Econômico, Rio de Janeiro, n. 166, 1990. Apresentado na CONFERENCE OF THE HISTORY OF ECONOMICS SOCIETY, 18., 1991, Maryland. Abstracts... Mariland: University of Maryland, 1991. Abstract Collection, p. 91. ENCONTRO NACIONAL DE ECONOMIA, 19., 1991, Curitiba. Anais... Curitiba: ANPEC, 1991. v. 4, p. 69-94. Versão em português: A perspectiva da escolha pública e a inclinação institucionalista de Knight. Revista Brasileira de Economia, n. 50, p. 111-113, jan/mar. 1996.

The Indetermination of Senior. Cambridge: Clare Hall, University of Cambridge, 1990c. Mimeog. Ensaio Econômico, Rio de Janeiro, n. 167, 1990. ENCONTRO NACIONAL DE ECONOMIA, 18., 1990, Brasília. Anais... Brasília, ANPEC, 1990, v. 1, p. 221-238. Versão em português: A Indeterminação de Senior. Revista de Economia Política, n. 11, p. 70-88, out./dez. 1991. 
Aplicabilidade de Teorias Econômicas: a Indeterminação de Senior. Projeto aprovado na seleção do PNPE-1990, IPEA, 30 nov. 1990 (Pré-projeto 30, set. 1990).

.Aplicabilidade de Teorias: Micro-Neoclássica e Estratégia Empresarial. Revista de Economia Política, n. 14, p. 53-76, abr./jun. 1994. Ensaio Econômico, n. 192, maio 1992.

.Memorial. Escrito para atender requisito dos concursos públicos de professor titular dos departamentos de Economia da Universidade de Brasília, em julho de 1992, e da Universidade Federal do Rio de Janeiro, em junho de 1993.

Teorias econômicas: a meia-verdade temporária. Revista Brasileira de Economia, n. 48, p. 203-216, abr./jun. 1994. Ensaio Econômico, Rio de Janeiro, n. 204, dez. 1992. Apresentado no congresso CAOS, ACASO E DETERMINISMO NAS CIÊNCIAS ARTES E FILOSOFIA, 1992, Rio de Janeiro. Anais... Rio de Janeiro: Câmara de Estudos Avançados do Forum de Ciência da UFRJ, 1992.

. The Ricardian Vice and The Indetermination of Senior. Ensaio Econômico, Rio de Janeiro, n. 205, dez. 1992. Apresentado na FIFTH ANNUAL INTERNATIONAL CONFERENCE OF THE SOCIETY FOR ADVANCEMENT OF SOCIO-ECONOMICS - SASE, 5., 1993, New York. Anais... New York: New School for Social Research, 1993.

Senior, Wagner and Schmoller: indetermination and social policy conclusions. In: BACKHAUS, J. G. (Ed.). Essays on Social Security and Taxation: Gustav von Schmoller and Adolph Wagner reconsidered. Marburg: Metropolis-Verlag, 1997. p. 361-386. A primeira versão, The Indetermination of Senior (ou The Indetermination of Wagner) and Schmoller as a social economist, foi apresentada na CONFERENCE ON SCHMOLLER AND WAGNER, 1992, Heilbronn. Abstracts... Heilbronn, Germany, Oct. 1992; circulando ainda em Ensaio Econômico, Rio de Janeiro, n. 212, mar. 1993. Versão em português: Wagner e Schmoller sob a luz da Indeterminação de Senior. Estudos Econômicos, n. 23, p. 319-345, maio/ago. 1993. ENCONTRO NACIONAL DE ECONOMIA, 21., 1993, Belo Horizonte. Anais... Belo Horizonte: ANPEC, 1993. v. 2, p. 421-438.

A Indeterminação de Senior como programa de pesquisa. Com revisões sucessivas, circulou em Estudos sobre a Indeterminação de Senior: exemplares para a iniciação científica. Ensaio Econômico, v. 1, n. 223, out. 1993; Ensaio Econômico, v. 2, n. 230, fev. 1994; Ensaio Econômico, n. 327, jun. 1998; em vários projetos de pesquisa, como CNPq, fev. 1995; UFRJ (CEPG/SAG 340201P066-2), 01 dez. 1995; DAAD/CAPES, abr. 1998.

; FERNANDEZ, B. P. M. O economista Maquiavel. CAOS, ACASO E CAUSALIDADE NAS CIÊNCIAS, ARTES E FILOSOFIA, 1993, Rio de Janeiro. Anais... Rio de Janeiro: Câmara de Estudos Avançados do Fórum de Ciência e Cultura da UFRJ, 1993. Ensaio Econômico, Rio de Janeiro, n. 230, p. 33-43, fev. 1994. Versão em inglês: The economist Machiavelli. Apresentado na SIXTH ANNUAL INTERNATIONAL CONFERENCE ON SOCIO-ECONOMICS - SASE, 6., 1994, PARIS. Abstracts... Paris: Hautes Études Commerciales School of Management, 1994. p. $50-51$.

Ser-e-não-ser. Rio de Janeiro: FEA/UFRJ, 1994. Mimeog.

. A Indeterminação de Senior e o currículo mínimo de Economia. Revista de Economia Política, n. 19, p. 182-199, jan./mar. 1999. Ensaio Econômico, Rio de Janeiro, n. 308, p. 1-30, jul. 1997. A versão embrionária, Por uma ciência econômica filosoficamente informada: a 
Indeterminação de Senior, foi apresentada no II ENCONTRO DOS ECONOMISTAS DE LÍNGUA PORTUGUESA, 2., 1995, Rio de Janeiro. Anais... Rio de Janeiro: IEI/UFRJ, 1995, v. 1, p. 9-25. Ensaio Econômico, Rio de Janeiro, n. 271, p. 1-19, out.

O espírito da Resolução 84. I SIMPÓSIO DO ENSINO DE ECONOMIA, 1., 1996, Rio de Janeiro. O ensino de Economia: questões teóricas e empíricas. Rio de Janeiro: CORECON/ RJ - IERJ - SINDECON, 1996.

Para onde vai a formação do economista: hegemonia ou pluralidade? In: LOUREIRO, M. R. (Org.). 50 anos de ciência econômica no Brasil. Petrópolis: Vozes / FIPE-USP, 1997. p. 283289. Apresentado no seminário BALANÇO DE TRÊS DÉCADAS DE CIÊNCIA ECONÔMICA NO BRASIL, 1., 1996, São Paulo. Anais... São Paulo: FEA/USP, 1996.

Avaliação do desempenho acadêmico: globalização e Trópico. Revista de Ciência e Tecnologia, v. 2, n. 1, p. 148-162, 1998 (RECITEC http://www.fundaj.gov.br). Texto para Discussão, PIMES/UFPE, n. 397, p. 1-11, set. 1997. Apresentado no SEMINÁRIO DE TROPICOLOGIA DA FUNDAÇÃO JOAQUIM NABUCO, 1., 1997, Recife. Anais... Recife: PIMES/UFPE, 1997 (núcleo temático Trópico e globalização); em Aulas Inaugurais no PIMES/ UFPE, Recife, 10 mar. 1997 e no Mestrado em Economia da UFF, 15 ago. 1997.

. Emulatig North American Economics: the education for the Ricardian Vice. Stuttgart: Institut für Volkswirtschaftslehre, Universität Hohenheim, 1998. Mimeog. Apresentado em seminários de pesquisa na Universität Hohenheim (Institut für Volkswirtschaftslehre, 12 nov. 1998) e na Freie Universität Berlin (John F. Kennedy-Institut, 1 dez. 1998 e LAI, 2 dez. 1998). (Em inglês apenas, incompleto).

\section{TRABALHOS ORIENTADOS}

AMARAL, S. L. Da Indeterminação de Senior para os juízos de valor: busca das raízes do desvio no pensamento de cairnes. Rio de Janeiro, 1995. Monografia (Conclusão de Curso) FEA/Universidade Federal do Rio de Janeiro.

ARAÚJO JR., E. A. Ensaios metodológicos sobre Ronald Coase: teoria da firma e das instituições jurídicas. Rio de Janeiro, 1996. Tese (Mestrado) - EPGE/Fundação Getúlio Vargas.

BARBOSA, M. C.; OLIVEIRA, E. de. A determinação de Hayek. CAOS, ACASO E CAUSALIDADE NAS CIÊNCIAS, ARTES E FILOSOFIA, 1993, Rio de Janeiro. Anais... Rio de Janeiro: Câmara de Estudos Avançados do Fórum de Ciência e Cultura da UFRJ, 1993.

BARBOSA, N. et al. Indeterminismo e a metodologia da Economia Positiva. Revista Brasileira de Economia, n. 48, p. 217-222, abr./jun. 1994. Apresentado no congresso CAOS, ACASO E DETERMINISMO NAS CIÊNCIAS, ARTES E FILOSOFIA, 1992, Rio de Janeiro. Anais... Rio de Janeiro: Câmara de Estudos Avançados do Fórum de Ciência e Cultura da UFRJ, 1992. Ensaio Econômico, Rio de Janeiro, n. 223, p. 27-32, out. 1993.

DATZ, M. O Vício Ricardiano em Gary Becker. Apresentado no congresso CAOS, ACASO E CAUSALIDADE NAS CIÊNCIAS, ARTES E FILOSOFIA, 1993, Rio de Janeiro. Anais... Rio de Janeiro: Câmara de Estudos Avançados do Fórum de Ciência e Cultura da UFRJ, 1993. 
FARIAS, W. J. Furtado à luz da Indeterminação de Senior. Rio de Janeiro, 1996. Monografia (Graduação) - IE/Universidade Federal do Rio de Janeiro.

FERNANDES, B. P. M. Maquiavel e o Vício Ricardiano. Rio de Janeiro: EPGE/Fundação Getúlio Vargas, 1993. Pesquisa em Bolsa de Aperfeiçoamento do CNPq. Mimeog.

FREITAS, F.; LOPES, C. F. L. R. Fragilidade financeira e instalibilidade estrutural. Apresentando no congresso CAOS, ACASO E CAUSALIDADE NAS CIÊNCIAS, ARTES E FILOSOFIA, 1993, Rio de Janeiro. Anais... Rio de Janeiro: Câmara de Estudos Avançados do Fórum de Ciências e Cultura da UFRJ, 1993.

FUKS, M. O paradigma entrópico na Economia: incerteza relativa aos recursos naturais. Revista Brasileira de Economia, n. 48, p. 223-229, abr./jun. 1994. Apresentado no congresso CAOS, ACASO E NAS CIÊNCIAS, ARTES E FILOSOFIA, 1992, Rio de Janeiro. Anais... Rio de Janeiro: Câmara de Estudos Avançados do Fórum de Ciência e Cultura da UFRJ, 1992. Derivado de Considerações preliminares sobre a Introdução do Conceito de Entropia na Ciência Econômica. Rio de Janeiro, 1992. Tese (Mestrado) - EPGE/Fundação Getúlio Vargas.

DUARTE, M. T. G. Teoria evolucionária e estratégia empresarial segundo a Indeterminação de Senior. Apresentado no congresso CAOS, ACASO E CAUSALIDADE NAS CIÊNCIAS, ARTES E FILOSOFIA, 1993, Rio de Janeiro. Anais... Rio de Janeiro: Câmara de Estudos Avançados do Fórum de Ciência e Cultura da UFRJ, 1993. Ensaio Econômico, Rio de Janeiro, n. 230, p. 2832, fev. 1994

Uma abordagem de desenvolvimento econômico: Kuznets sob análise da Indeterminação de Senior. Rio de Janeiro, 1994. Tese (Mestrado) - IEI/Universidade Federal do Rio de Janeiro.

GRUMBACH, S. Weber e a Indeterminação de Senior. Apresentado no congresso CAOS, ACASO E CAUSALIDADE NAS CIÊNCIAS, ARTES E FILOSOFIA, 1993, Rio de Janeiro. Anais... Rio de Janeiro: Câmara de Estudos Avançados do Fórum de Ciência e Cultura da UFRJ, 1993. Ensaio Econômico, Rio de Janeiro, n. 230, p. 23-27, fev. 1994. Desenvolvido em Críticas a cientificismo. Rio de Janeiro, 1994. Monografia (Graduação) - FEA/Universidade Federal do Rio de Janeiro.

. Filosofia da ciência e metodologia econômica: um estudo crítico. Rio de Janeiro, 1997. Tese (Mestrado) - IE/ Universidade Federal do Rio de Janeiro.

LIBÂNIO, G. de A. Uma nota sobre Lucas e o Vício Ricardiano. Revista de Economia Política, n. 17. p. 140-146, out./dez. 1997.

LOPES, C. F. L. R. Mises e o Vício Ricardiano. Revista Brasileira de Economia, n. 48 p. 231234, abr./jun. 1994. Ensanio Econômico, Rio de Janeiro, n. 223, p. 17-21, out. 1993. Derivado de Mises e a Indeterminação de Senior. Rio de Janeiro, 1992. Monografia (Graduação) - FEA/ Universidade Federal do Rio de Janeiro.

O método em Minsky: uma aborgadem segundo a Indeterminação de Senior: Rio de Janeiro, 1995. Tese (Mestrado) - IEI/Universidade Federal do Rio de Janeiro.

MELlO, C. M. B. A Indeterminação de Senior e a teoria behaviorista da decisão. Rio de Janeiro, 1994. Monografia (Graduação) - FEA/Universidade Federal do Rio de Janeiro. Bolsa de Iniciação Científica do CNPq. 
MELLO, V. R. de. O realismo dos pressupostos em Economia. Rio de Janeiro: Editora da FGV, 1990. EPGE, mimeog.

MONTEIRO, M. B. Marshall e a Indeterminação de Senior. Apresentado no congresso CAOS, ACASO E NAS CIÊNCIAS, ARTES E FILOSOFIA, 1992, Rio de Janeiro. Anais... Rio de Janeiro: Câmara de Estudos Avançados do Fórum de Ciência e Cultura da UFRJ, 1992. Ensaio Econômico, Rio de Janeiro, n. 223, p. 33-40, out. 1993.

MULS, L. M. O Vício Ricardiano no debate sobre o processo de trabalho. Apresentado no congresso CAOS, ACASO E CAUSALIDADE NAS CIÊNCIAS, ARTES E FILOSOFIA, 1993, Rio de Janeiro. Anais... Rio de Janeiro: Câmara de Estudos Avançados do Fórum de Ciência e Cultura da UFRJ, 1993, Rio de Janeiro. Anais... Rio de Janeiro: Câmara de Estudos Avançados do Fórum de Ciência e Cultura da UFRJ, 1993. Versão aperfeiçoada para o II ENCONTRO DOS ECONOMISTAS DE LÍNGUA PORTUGUESA, 2., 1995, Rio de Janeiro. Anais... Rio de Janeiro: IEI/UFRJ, 1995, v. 1, p. 26-34.

NEVES, S. E. B. John Stuart Mill e a Indeterminação de Senior: a interdisciplinaridade da Ciência Aplicada e o campo de estudo da Economia Social. Rio de Janeiro, 1996. Monografia (Graduação) - FEA/Universidade Federal do Rio de Janeiro.

OREIRO, J. L. Determinismo, ordem e ciclo econômico em Shackle. (Bolsa de Iniciação Científica do CNPq.). Apresentado no congresso CAOS, ACASO E CAUSALIDADE NAS CIÊNCIAS, ARTES E FILOSOFIA, 1993, Rio de Janeiro. Anais... Rio de Janeiro: Câmara de Estudos Avançados do Fórum de Ciência e Cultura da UFRJ, 1993.

ORIND, F. Keynes, incerteza e Indeterminação de Senior. Rio de Janeiro, 1995. Monografia (Graduação) - FEA/Universidade Federal do Rio de Janeiro.

RAMOS, E. A. de A. A revolução em finanças sob uma perspectiva metodológica. Rio de Janeiro, 1995. Tese (Mestrado) - EPGE/Fundação Getúlio Vargas.

VERNENGO, M. A macro como disciplina autônoma. Apresentado no congresso CAOS, ACASO E CAUSALIDADE NAS CIÊNCIAS, ARTES E FILOSOFIA, 1993, Rio de Janeiro. Anais... Rio de Janeiro: Câmara de Estudos Avançados do Fórum de Ciência e Cultura da UFRJ, 1993.

. Keynes e o Vício Ricardiano. Revista de Economia política, n. 14, p. 129-133, jul./set. 1994. Ensaio Econômico, Rio de Janeiro, n. 223, p. 22-26, out. 1994.

. Método, moeda e demanda efetiva: algumas considerações sobre a posição de Keynes na história do pensamento econômico. Rio de Janeiro, 1995. Tese (Mestrado) - IEI/Universidade Federal do Rio de Janeiro.

\section{TRABALHOS PRECURSORES}

SILVEIRA, A. M. da. A Universidade e o Plano Básico de Desenvolvimento Científico e Tecnológico: crítica. Ciência e Cultura, n. 26, p. 249-251, mar. 1974. 
. Brasil 77: variações sobre a conjuntura econômica. Debate Econômico, v. 1, p. 3-6, maio 1978. Reproduzido em (Org.). Debate Econômico. Rio de Janeiro: Multiplic, 1981.

Magistério, moral e política econômica. In: (Org.). Moeda e redistribuição de renda. Rio de Janeiro: Multiplic, 1981.

Racionalidade e pedra fundamental: variações sobre a tese de E. Coelho. REUNIÃO NACIONAL DA ANPAD, 4., 1980, Natal. Anais... Natal, 1980. p. 249-258.

. SIMON e o satisfaziamento. Literatura Econômica, n. 5, p. 587-606, set./out. 1983. maio 1984.

Indexação e ambiência geral de negócios. Ensaio Econômico, Rio de Janeiro, n. 42,

. A Indeterminação de Morgenstern. Revista Brasileira de Economia, n. 38, p. 357-383, out./dez 1984. ENCONTRO NACIONAL DE ECONOMIA, 12., 1984, São Paulo. Anais... São Paulo: ANPEC, 1984. v. 1, p. 388-418, em sua primeira versão, Natureza dos dados econômicos: ad usum delphini.

. Interpretação econômica, inflação e indexação. In: BIANCHI, A. M. F. (Org.). Questões de método na ciência econômica. São Paulo: IPE/USP, 1986. Ensaio Econômico, Rio de Janeiro, n. 61, maio 1985. 1986.

Os dados econômicos e a Nova República. Análise e conjuntura, p. 157-163, set./dez.

Assessoria econômica para a estratégia de Governos Estaduais: elaborações sobre uma estrutura aberta. In: (Org.). Diagnóstico macroeconômico e outros ensaios. Belo Horizonte: Fundação João Pinheiro, 1986. ENCONTRO NACIONAL DE ECONOMIA, 14., 1986, Brasília. Anais... Brasília: ANPEC, 1986. v. 1, p. 395-411.

Filosofia e política econômica: o Brasil do autoritarismo. 2. ed. Rio de Janeiro: IPEA, 1992. O projeto, aprovado na seleção do PNPE-1984 (agosto), teve como embrião uma pesquisa realizada em 1983 e divulgada no ensaio de maio de 1984, listado acima. Completado e entregue ao IPEA/INPES em novembro de 1985, circulou em publicações parciais como "Inflação e indexação: o conflito redistributivo, Rio de Janeiro, EPGE, fevereiro de 1985 (Relatório de Pesquisa para o PNPE), e "Limitações da Economia e dos economistas: o Brasil do autoritarismo, Ensaio número 1 do CMA, Belo Horizonte, FACE/UFMG, agosto de 1985.

Keynes na sedição da escolha pública. Análise Econômica, n. 6, p. 23-27, nov. 1987 / mar. 1988. Apresentado no encontro "Keynes em debate", Porto Alegre, IEPE/UFRS, 27-28 ago. 1987.

Modelos do homem: economia e administração. In: CARVALHO, A. O. Administração contemporânea: algumas reflexões. Belo Horizonte: Editora da UFMG, 1988. 


\section{REFERÊNCIAS}

ALCHIAN, A. A. Uncertainty, evolution and economic theory. Journal of Political Economy, v. 58, p. 211-221, June 1950. (EM II: p. 249-265, Aug. 1982).

ANSOFF, H. I. Corporate strategy. New York: McGraw-Hill/Harmondsworth, 1965; Middlesex: Penguim Books, 1987.

. Strategic management. London: Macmillan, 1979.

BARANZZINI, M.; SCAZZIERI, R. Knowledge in economics: a framework. In:

(Ed.). Foundations of economics: structure of inquiry in economic theory. New York: Basil Balckwell, 1986.

BECKER, G. S. Irrational Behavior and economic theory. Journal of Political Economy, v. 70, p. 1-13, Feb. 1962. (EM II: p. 267-287, Aug. 1982).

BIANCHINI, A. M. A pré história da Economia: de Maquiavel a Adam Smith. São Paulo: Hucitec, 1988.

BLAUG, M. The methodology of econnomics: or how economists explain. Cambridge: Cambridge University Press, 1981.

Great economists since Keynes. Brighton, Sussex: Harvester Press, 1985.

BRUNNER, K. Knowledge, values and the choice of economic organization. Kyklos, v. 23, Fasc. 3, p. 558-579, 1970. (EM II: p. 1-21, Oct. 1981).

Reflections on the political economy of Government: the persistent growth of Government, Schweizeriche Zeitschrift Für Volkswirtschaft Und Statistik, Heft 3, p. 649-680, 1978. (EM II: p. 45-86, Okt. 1981).

. MECKLING, W. H. The perception of man and the conception of Government. Journal of Money, Credit and Banking, v. 9, p. 70-85, Feb. 1977. (EM II: p. 23-44, Oct. 1981).

BUCHANAN, J. M. Liberty, market and the State: political enonomy in the 1980s. Washington Square: New York University Press, 1985.

. The constitution of economic policy. In: Lex Prix Nobelen 1986. Stockholm: Nobel Foundation, 1987.

. The economic theory of politics reborn. Challenge, n. 31, p. 4-10, Mar./Apr. 1988. 1986.

; TOLLISON, R. D. A theory of truth in autobiography. Kyklos, n. 39, Fasc. 4, p. 507-517,

; TULLOCK, G. The calculus of consent: logical foundations of constitutional democracy. Michigan: University of Michigan Press, 1965.

CALDWELL, B. Beyond positivism: economic methodology in the twentieth century. London: George Allen \& Unwin, 1985. 
; COATS, A. W. the retoric of economists: a comment on McCloskey. Journal of Economic Literature, v. 22, p. 575-578, June 1984.

CARABELLI, A. On Keynes Method. London: MacMillan, 1988.

CASTRO, C. de M. A prática da pesquisa. São Paulo: McGraw-Hill do Brasil, 1977.

COASE, R. H. The insitutional structure of production. In: Les Prix Nobel en 1991. Stockholm: Nobel Foundation, 1992.

COLANDER, D.; COATS, A. W. (Ed.). The spread of economic ideas. Cambridge: Cambridge University Press, 1989.

COMIM, F. V. Probabilidade e conduta racional em Keynes. São Paulo: USP, Dep. Economia, 1993. Mimeog.

DASGUPTA, A. K. Epochs of economic theory. New York: Basil Balckwell, 1987.

DEANE, P. The scope and method of economic theory. Economic Journal, n. 95, p. 909-927, Dec. 1983.

DEBREU, G. Discours des laureats. In: Les Prix Nobel en 1983. Stockholm: Nobel Foundation, 1984.

Mar. 1991.

The mathematization of economic theory. American Economic Review, n. 81, p. 1-7,

FEYERABEND, P. K. Against Method. Minnesota Studies in the Philosophy of Science, Minnesota, n. 4, 1970.

FRANK, R. H. et al. Does studing economics inhibit cooperation? Journal of Economic Perspectives, n. 7, p. 159-171, 1993.

FREY, B. S. et al. Consensus and dissension among economist: an empirical inquiry. American Economic Review, n. 74, p. 986-994, Dec. 1984.

FRIEDMAN, M. The methodology of positive economics. In: Essays in positive economics. Chicago: University of Chicago Press, 1953. (EM I: p. 163-200, Feb. 1981).

FRISCH, R. From utopian theory to practical applications: the case of econometrics. In: Les Prix Nobel en 1969. Stockholm: Nobel Foundation, 1970. (Revista Brasileira de Economia, n. 39, p. 109-140, jan./mar. 1985).

. Energy and economic myths. Southern Economic Journal, n. 41, p. 347-381, Jan. 1975. (EM I: p. 337-389, Aug. 1981).

Methods in economic science. Journal of Economic Issues, v. 13, p. 317-328, June 1979. (EM I: p. 115-127, Dec. 1980).

GROENEWEGEN, P. D. (Ed.). Alfred Marshall on the method and History of Economics. Sydney: Center for the Study of the History of Economic Thought, 1990.

HAHN, F. Equilibrium and macroeconomics. Oxford: Basil Blackwell, 1984. 
HARRIS, S. E. A postscript by the editor. In: SAMUELSON, P. A. et al. Mathematics in economics: discussion of Mr. Novick's article. Review of Economics and Statistics, v. 36, p. 382-386, Nov. 1954, (EM I: p. 106-113, Dec. 1980).

HASHELL, T. L. (Ed.) The authority of experts: studies in History and theory. Bloomington: Indiana University Press. 1984.

HAYEK, F. A. von. Law, Legislation and liberty, I: rules and order. Chicago: University onf Chicago Press, 1973.

The pretense of knowledge. In: Les Prix Nobel en 1974. Stockholm: Nobel Foundation, 1975. (EM II: p. 183-193, Apr. 1982; Revista Brasileira de Economia, n. 37, p. 513-522, out./ dez. 1983).

Law, legislation and liberty, II: the mirage of Social Justice. Chicago: University of Chicago Press, 1978.

Law, legislation and liberty, III: the political order of a free people. Chicago: University of Chicago Press. 1981.

HEER, F. The medieval world. London: Weidenfeld \& Nicolson, 1969. (EM II: p. 305-367, Oct. 1982, Capítulos 9-12).

HEGENBERG, L. Introdução à filosofia da ciência. São Paulo: Herder, 1965.

HEILBRONER, R. L. The Worldly philosophers. New York: Simon \& Schuster, 1986.

HEISENBERG, W. Phisics and philosophy. London: George Allen \& Unwin, 1963.

. A parte e o todo. Rio de Janeiro: Contraponto, 1996.

HICKS, J. R. Some questions of time in economics. In: TANG, A. M. et al. Evolution, welfare, and time in economics: essays in honor of Georgescu-Roegen. Lexington, MA: Lexington Books, 1976.

Revolutions in Economics. In: LATSIS, S. J. (Ed.). Methods and appraisal in economics. Cambridge: Cambridge University Press, 1980.

HUTCHISON, T. W. The politics and philosophy of economics. Oxford: Basil Blackell, 1981.

JOHNSON, H. G. The keynesian revolution and the monetarist counter-revolution. American Economic Review, v. 61, p. 145-162, May 1971. (EM I: p. 145-162, Feb. 1981).

KANTOROVICH, L. V. Mathematics in economics: achievements, difficulties, perspectives. In: Les Prix Nobel en 1975. Stockholm: Nobel Foundation, 1976. Revista Brasileira de Economia, n. 38, p. 275-284, jul./set. 1984).

KEYNES, J. M. Essays in biography. London: Rupert Hart-Davis, 1951.

Essays in persuasion. London: MacMillan / Cambridge University Press, 1984.

KNIGHT, F. H. Ethics and the Economic Interpretation. Quarterly Journal of Economics, v. 35, p. 454-481, 1922. Reproduced in KNIGHT, F. H. The ethics of competition and other essays. London: Allen \& Unwin, 1936. (EM I: p. 3-23, Oct. 1980). 
The ethics of competition. Quarterly Journal of Economics, v. 36, p. 579-624, 1923. Reproduced in _. The ethics of competition and other essays. London: Allen \& Unwin, 1936. (EM II: p. 87-118, Dec. 1981).

Intellingence and democratic action. Cambridge, MA: Harvard University Press, 1960.

KRUEGER, A. O. et al. Report of the Comm ission on Graduate Education in Economics. Journal of Economic Literature, v. 29, p. 1035-1053, Sept. 1991.

KUHN, T. S. The structure of scientific revolutions. Chicago: University of Chicago Press, 1971.

Logic of discovery or psychology of research. In: LAKATOS, I.; MUSGRAVE, A. Cristicism and the growth of knowledge. Cambridge: Cambridge University Press, 1980.

KUZNETS, S. Six lectures on economic growth. New York: Free Press of Glencoe, 1961.

Modern economic growth: findings and reflections. In: Lex Prix Nobel en 1971. Stockholm: Nobel Foundation, 1972. (Revista Brasileira de Economia, n.39, p. 225-239, abri./ jun. 1985).

LACHMANN, L. M. Macro-economic thinking and the market economy: an essay on the neglect of the micro-foundations and its consequences. London: The Institute of Economic Affairs, 1973.

LAKATOS, I.; MUSGRAVE, A. (Ed.). Criticism and the growth of knowledge. Cambridge University Press, 1980.

LANGE, O. The scope and method of economics. Review of Economic Studies, v. 13, p. 19-32, 1947 (EM II: p. 119-138, Dec. 1981).

LAWSON, T. Uncertainty and economic analysis. Economic Journal, n. 95, p. 909-927, Dec. 1985.

Abstraction, tendencies and stylized facts: a realist approach to economic analysis. Cambridge Journal of Economics, n. 13, p. 59-78, 1989.

. et al. Kaldor's contribution to economics: an introduction. Cambrige Journal of Economics, n. 13, p. 1-8, 1989.

LEIJONHUFVUD, A. Life among the Economists. Western Economic Journal, p. 327-337, Sept. 1973. (EM II: p. 169-181, Dec. 1981).

MACHLUP, F. Methodology of economics and other social sciences. New York: Academic Press, 1978.

MARCH, J. G. Bounded rationality, ambiguity and engineering of choice. Bell Journal of Economics, n. 9, p. 587-608, 1978. (EM II: p. 195-225, Apr. 1982).

; HERBERT A. S. Organizations. New York: John Wiley \& Sons, 1959.

MARGENAU, H. What is a Theory? In: KRUPP, S. R. (Ed.) The structure of Economic Science. Englewood, NY: Prentice-Hall, 1966. (EM II: p. 289-304, Aug. 1982). 
MASTERMAN, M. The nature of a paradigm. In: LAKATOS, I.; MUSGRAVE, A. (Ed.). Cristicism and the growth of knowledge. Cambridge: Cambridge University Press, 1980.

MCCLOSKEY, D. N. The rhetoric of economics. Journal of Economic Literature, v. 21, p. 481517, June 1983

MELTZER, A. H. Limits of short-run stabilization policy. Economic Inquiry, v. 25, p. 1-14, Jan. 1987.

MENDES, A. D. Novo currículo mínimo de Ciências Econômicas: parecer. Documenta, n. 282, p. 163-193, Jun. 1984. Reproduzido em Revista ANPEC, n. 2, p. 203-239, nov. 1997.

MILL, J. S. Essays on some unsettled questions of political economy. London: Longmans, Green, and Co., 1877.

MORGENSTERN, O. On the accuracy of economic observations. Princeton, NJ: Princeton University Press, 1963.

MORRIS-SUZUKI, T. A history of Japanese economic thought. London: Routledge, 1991.

MYRDAL, G. Objectivity in Social Research. New York: Pantheon, 1969.

NAGEL, E. Assumptions in economic theory. American Economic Review, v. 53, p. 211-219, May 1963. (EM II: p. 227-236, Apr. 1982).

NEVILLE KEYNES, J. The scope and method of political economy. London: MacMillan, 1904.

NOVICK, D. Mathematics: logic, quantity, and method. Review of Economics and Statistics, v. 36, p. 357-358, Nov. 1954. (EM I: p. 61-64, Dec. 1980).

OHLIM, B. Speech on the Nobel Laureate. In: Les Prix Nobel en 1971. Stockhlm: Nobel Foundation, 1972.

PASINETTI, L. L. Theory of Value: a source of alternative paradigms in economic analysis. In: BARANZZINI, M.; SCAZZIERI, R. (Ed.) Foundations of economics: structures of inquiry in economic theory. New York: Basil Blackwell, 1986.

PATINKIN, D. The Chicago tradition, the quantity theory, and Friedman. Journal of Money, Credit and Banking, v. 1, p. 46-70, Feb. 1969. (EM II: p. 139-167, Dec. 1981).

POPPER, K. R. The logic of scientific discovery. New York: Sciences Editions, 1961.

Conjectures and refutations. New York: Basic Books, 1965.

Autobiografia intelectual. São Paulo: Cultrix, 1976.

Lógica das Ciências Sociais. Rio de Janeiro: Tempo Brasileiro/UnB, 1978.

Normal science and its danger. In: LAKATOS, I.; MUSGRAVE, A. Cristicism and the growth of knowledge. Cambridge: Cambridge University Press, 1980.

PRICE, D. K. The Scientific State. Cambridge, MA: Harvard University Press, 1965.

PRIGOGINE, I.; STENGERS, I. A nova aliança: metamorfose da ciência. Brasília: Editora $\mathrm{UnB}, 1991$. 
REGO, J. M. (Org.). Retórica na Economia. São Paulo: Editora 34, 1996.

REID JR., J. D. The Theory of Sharecropping: Occam's razor and economic analysis. History of Political Economy, n. 19, p. 551-569, 1987.

ROBINSON, J. The second crisis of economic theory. American Economic Review, v. 62, p. 110, May 1972.

. Economic philosophy. Middlesex, England: Penguin Books, 1983.

ROLL, E. A history of economic thought. London: ff - faber and faber, 1987.

SAMUELS, W. J. The methodology of economics and the case for policy diffidence and restraint. Review of Social Economy, v. 47, p. 113-133, 1989.

SAMUELSON, P. A. Comments to professor Nagel's article. American Economic Review, v. 53, p. 231, May 1963. (EM II: p. 240-247, Apr. 1982).

. Maximum principles in analytical economics. In: Les Prix Nobel en 1970. Stocknolm: Nobel Foundation, 1971. (Revista Brasileira de Economia, n. 38, p. 401-419, out./dez. 1984).

; KLEIN, L. R. et al. Mathematics in economics: discussion of Mr. Novick's article. Review of Economics and Statistics, v. 36, p. 359-386, Nov. 1954. (EM I: p. 65-113, Dec. 1980).

SCHUMPETER, J. A. Science and ideology. American Economic Review, v. 39, p. 345-359, Mar. 1949. (Em I: p. 129-144, Feb. 1981).

. The theory of economic development. Cambridge, MA: Harvard University Press, 1959. History of economic analysis. London: Allen \& Unwin, 1986.

SENIOR, N. W. An outline of the theory of political economy. London: Kimble \& Bradford, 1938.

SIMON, H. A. Administrative Behavior. New York: MacMillan, 1947.

A Behavioral model of rational choice. Quarterly Journal of Economics, n. 69, p. 99$118,1995$.

. Models of man. London: Chapmam \& Hall, 1957.

Comments to professor Nagel's article. American Economic Review, v. 53, p. 229-231, May 1963. (EM II: p.237-239, Apr. 1982).

On judging the plausibility of theories. In: VON ROOTSELAAR; STAAL (Ed.). Logic, method, and philosophy of sciences. Amsterdam: North Holland, 1968.

Rational decision making in business organizations. In: Les Prix Nobel, en 1978. Stockholm: Nobel Foundation, 1979. (EMI: p. 25-60, Oct. 1980; Revista Brasileira de Economia, n. 38, p. 111-142, jan./mar. 1984).

Models of my life. U.S.A.: Basic Books, 1991. 
STIGLER, G. F. The Influence of events an policies in economic theory. American Economic Review, v. 50, p. 36-45, May 1960.

The process and progress of economics. In: Les Prix Nobel en 1982. Stockholm: Nobel Foundation, 1983.

TINGERGEN, J. The use of models: experience and prospects. In: Les Prix Nobel en 1969. Stockholm: Nobel Foundation, 1970. (Revista Brasileira de Economia, n. 37, p. 375-385, jul./ set. 1983).

TOYNBEE, A. J. A study of History. New York: Oxford University Press, 1962. Mankind and mother earth. London: Oxford University Press, 1976.

WAGNER, A. Systematische Natinaleconomie. Jahrburcher fur Nationaleconomie und Statistik. Translated in the Quarterly Journal of Economics, v. 1, p. 113-133, 1886 ( Wagner on the presente state of political economy). 1981. Marshall's principies of economics. Quarterly Journal of Economics, v. 5, p. 319-338,

WEBER, M. The methodology of the social sciences. Glencoe, IL: Free Press, 1949.

Economy and society. In: ROTH, G.; WITTICH, C. (Ed.). Berkeley: University of Califonia Press, 1978. 